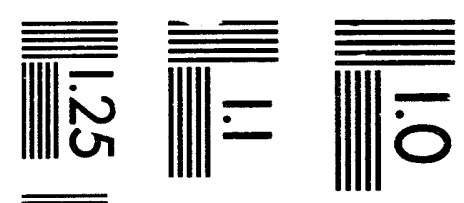

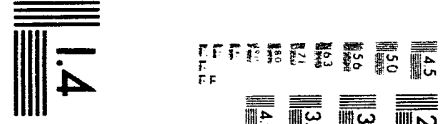

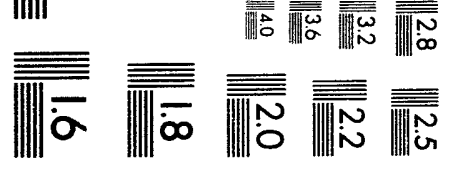



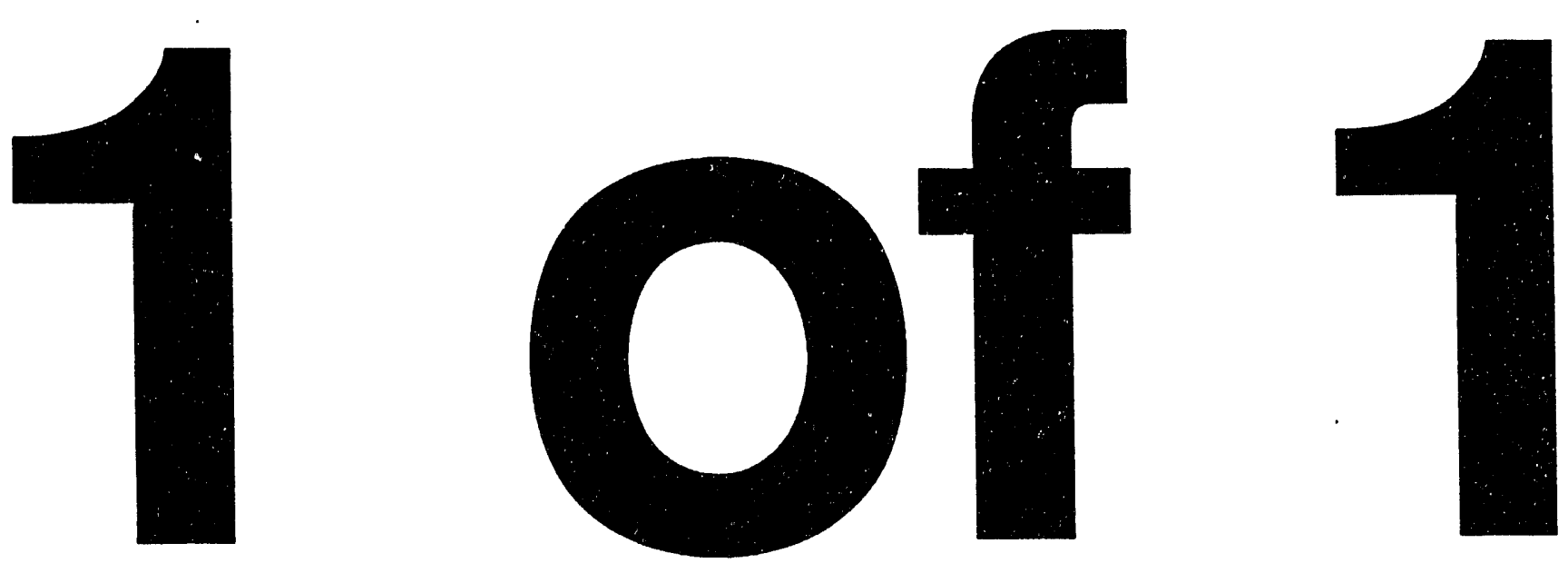


\title{
Conf-9310220--3
}

UCRL-JC-115601

PREPRINT

\section{A Concurrent, Multigroup, Discrete Ordinates Model of Neutron Transport}

\author{
M.R. Dorr \\ C. H. Still
}

This paper was prepared for submittal to the 1993 Scalable Parallel Libraries Conference Starkville, Mississippi

October 6-8, 1993

October 22, 1993

This is a preprint of a paper intended for publication in a journal or proceedings. Since changes may be made before publication, this preprint is made available with the understanding that it will not be cited or reproduced without the permission of the author.

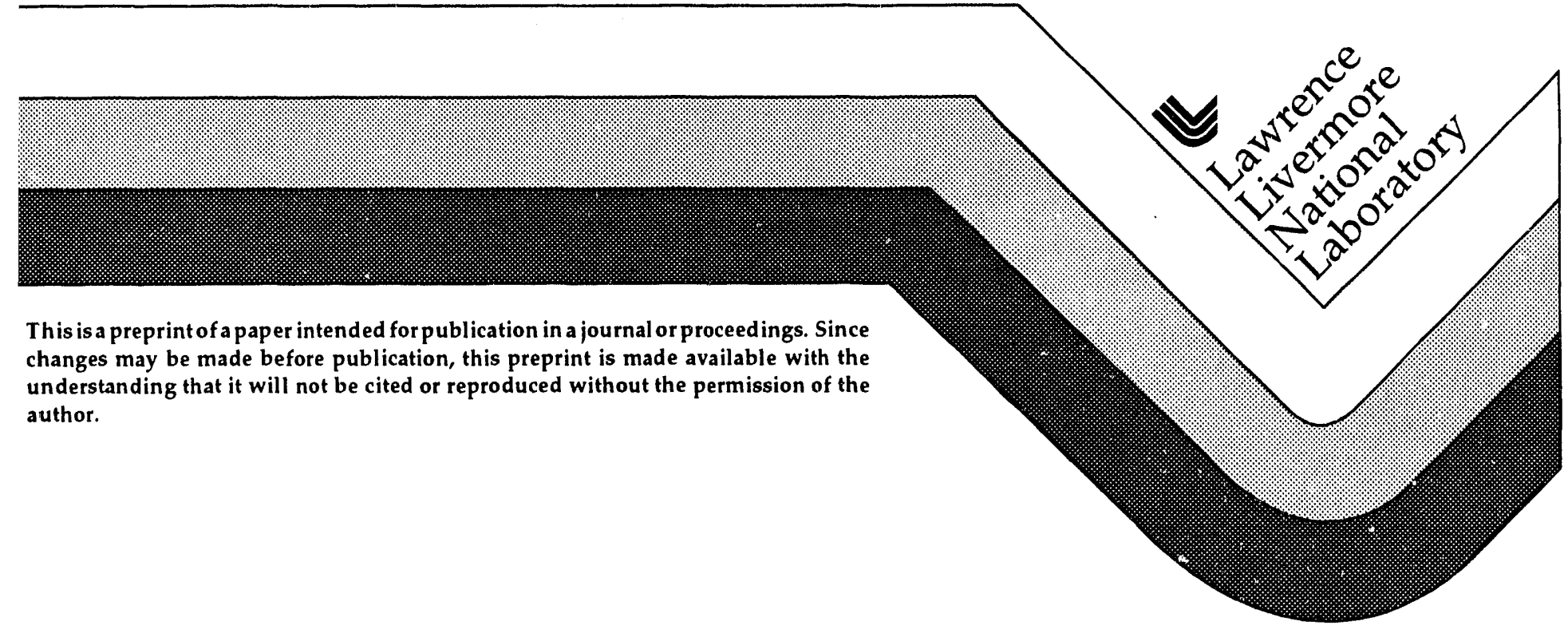




\section{DISCLAIMER}

This document was prepared as an account of work sponsored by an agency of the United States Goverument. Neither the United States Government nor the University of California nor any of their employees, makes any warranty, express or implied, or assumes any legal liability or responsibility for the accuracy, completeness, or usefulmess of any information, apparatus, product, or process disclosed, or represents that its use would not infringe privately owned rights. Reference herein to any specific commercial products, process, or service by trade name, trademark, manufacturer, or otherwise, does not necessarily constitute or imply its endorsement, recommendation. or favoring by the United States Government or the University of California. The views and opinions of authors expressed herein do not necessarily state or reflect those of the United States Government or the University of California, and shall not be used for advertising or product endorsement purposes. 


\section{A Concurrent, Multigroup, Discrete Ordinates Model of Neutron Transport *}

\author{
M. R. Dorr \\ Numerical Mathematics Group, L-316 \\ Lawrence Livermore National Laboratory \\ Livermore, CA 94550
}

\author{
C. H. Still \\ ICF Code Development Group, L-472 \\ Lawrence Livermore National Laboratory \\ Livermore, CA 94550
}

\begin{abstract}
We present an algorithm for the concurrent solution of the linear system arising from a multigroup, discrete ordinates model of neutron transport. The target architectures consist of distributed memory computers ranging from workstation clusters to massively parallel computers. Based on an analysis of the memory requirement and floating point complexity of matrixvector multiplication in the iterative solution of the linear system, we propose a data layout and communication strategy designed to achieve scalability with respect to all phase space variables. Numerical results are presented to demonstrate the performance of the algorithm on the $n C U B E / 2$.
\end{abstract}

\section{Introduction}

The ability to model the transport of neutrons through a background medium is important in several scientific and engineering activities. Among these are the design of both fission and fusion reactors, the planning of medical treatments, and the interpretation of nuclear well log data in oil and gas exploration. In this paper, we present an algorithm for the concurrent solution of the linear system arising from a multigroup, discrete ordinates model of neutron transport. The algorithm is designed to be implementable on a wide range of distributed memory computers ranging from workstation clusters to massively parallel computers. Such machines are characterized by some number of nodes (consisting of a processor and local memory) connected by a network. Communication among the nodes is achieved by the passing of messages through the network. The challenge of designing algorithms

- This work was performed under the auspices of the U.S Department of Energy by the Lawrence Livermore National Laboratory under contract W-7405-Eng-48. for such machines is that of deciding how data layout and communication should be managed in such a way as to minimize the time to solve the problem and to allow for scalability with respect to the important problem parameters. Scalability enables the flexible assignment of a fixed number of nodes to a variety of problem types, as well as the solution of larger problems by applying additional nodes.

In Section 2 we present the neutron transport equation and its discretization. A linear system is obtained whose concurrent solution is considered in Section 3. Based on an analysis of the memory requirement and floating point complexity of matrix-vector multiplication in the iterative solution of the linear system, we propose a data layout and communication strategy designed to achieve scalability with respect to all phase space variables. In Section 4 we briefly discuss an implementation of the algorithm, and in Section 5 we present some scalability results obtained on an nCUBE/ 2 .

\section{Problem formulation}

We consider the steady-state, neutron transport equation [1]

$$
\begin{aligned}
& \Omega \cdot \nabla \psi(x, E, \Omega)+\sigma(x, E) \psi(x, E, \Omega)= \\
& \int_{0}^{\infty} \sigma\left(x, E^{\prime} \rightarrow E\right) \int_{\mathcal{S}^{2}} \psi\left(x, E^{\prime}, \Omega^{\prime}\right) d \Omega^{\prime} d E^{\prime} \\
& +q(x, E, \Omega) \\
& x \in D, \quad 0<E<\infty, \quad \Omega \in \mathcal{S}^{2}, \\
& \psi(x, E, \Omega)=\psi^{\text {inc }}(x, E, \Omega), \quad x \in \partial D \\
& 0<E<\infty, \Omega \in \mathcal{S}^{2} \ni \Omega \cdot n_{\partial D}(x)<0
\end{aligned}
$$

where $D$ is a region in $\Re^{3}$ with boundary $\partial D, n_{\partial D}(x)$ is the outward pointing unit normal to $\partial D$ at $x$ and $\mathcal{S}^{2}$ is the unit sphere in $\Re^{3}$. Equation (1) models the 
transport of neutrons through an isotropically scattering background material. The unknown quantity $\psi(x, E, \Omega)$ represents the flux of neutrons at the spatial point $x$ moving in the direction $\Omega$ with energy $E$. The coefficients $\sigma(x, E)$ and $\sigma\left(x, E^{\prime} \rightarrow E\right)$ are the total and scattering cross-sections, respectively. The term $q(x, E, \Omega)$ represents an external, volumetric neutron source, while $\psi^{\text {inc }}(x, E, \Omega)$ represents a source of neutrons incident upon $\partial D$.

A semi-discretization of (1) can be obtained using a multigroup discretization of the energy $E$ and a discrete ordinates, or $S_{N}$, treatment of the neutron direction $\Omega$ (see, e.g., [2]). In the multigroup approach, the energy $E$ is restricted to a finite interval partitioned into subintervals, or "groups":

$$
E_{\max }=E_{0}>E_{1}>\cdots>E_{G}=E_{\min } .
$$

The equation (1) is then averaged over each group $E_{g}<E<E_{g-1}$ and the cross-sections $\sigma$ are approximated by certain "flux-weighted averages" to maintain linearity. The discrete ordinates approximation amounts to selecting a finite set of points $\Omega_{m}$, $m=1, \ldots, M$ on the unit sphere $\mathcal{S}^{2}$ and corresponding quadrature weights $w_{m}$, then collocating (1) at the $\Omega_{n}$ while making the approximation

$$
\int_{\mathcal{S}^{2}} \psi(x, E, \Omega) d \Omega \approx \sum_{m=1}^{M} w_{m} \psi\left(x, E, \Omega_{m}\right) .
$$

This yields the following semi-discretization of (1):

$$
\begin{aligned}
& \Omega_{m} \cdot \nabla \psi_{g, m}(x)+\sigma_{g}(x) \psi_{g, m}(x)= \\
& \sum_{g^{\prime}=1}^{G} \sigma_{g^{\prime} \rightarrow g}(x) \sum_{m^{\prime}=1}^{M} w_{m^{\prime}} \psi_{g^{\prime}, m^{\prime}}(x)+q_{g, m}(x), \\
& x \in D, \quad 1 \leq g \leq G, \quad 1 \leq m \leq M,
\end{aligned}
$$

$$
\begin{aligned}
\psi_{g, m}(x)=\psi_{g, m}^{\text {inc }}(x), \quad x \in \partial D, \quad 1 \leq g \leq G, \\
1 \leq m \leq M \quad \ni \Omega_{m} \cdot n_{\partial D}(x)<0 .
\end{aligned}
$$

We now wish to express (4) in operator notation. Let

$$
\mathcal{H}_{g}\left(\psi_{1}, \ldots, \psi_{M}\right)=\left(\mathcal{H}_{g, 1} \psi_{1}, \ldots, \mathcal{H}_{g, M} \psi_{M}\right)
$$

where

$$
\begin{array}{r}
\mathcal{H}_{g, m} \psi= \\
\left(\sigma_{g}^{-1}(x) \Omega_{m} \cdot \nabla \psi(x)+\psi(x),\left.\psi\right|_{x \in \partial D \ni n_{\theta D}(x) \cdot \Omega<0}\right)
\end{array}
$$

and define

$$
\left(\mathcal{L}\left(\psi_{1}, \ldots, \psi_{M}\right)\right)(x)=
$$

$$
\begin{gathered}
\sum_{m=1}^{M} w_{m} \psi_{m}(x) \\
\left(\mathcal{L}^{+} \phi\right)(x)=(\phi(x), \ldots, \phi(x)) \\
\left(\Gamma_{g, g^{\prime}} \phi\right)(x)=\sigma_{g}^{-1}(x) \sigma_{g^{\prime} \rightarrow g}(x) \phi(x) \\
\left(\mathcal{Z}\left(\psi_{1}, \ldots, \psi_{M}\right)\right)(x) \equiv \\
\left(\mathcal{Z}_{1} \psi_{1}(x), \ldots, \mathcal{Z}_{M} \psi_{M}(x)\right) \\
\equiv \quad\left(\left(\psi_{1}(x), 0\right), \ldots,\left(\psi_{M}(x), 0\right)\right) \\
\mathcal{Q}_{g}=\left(\left(\sigma_{g}^{-1} q_{g, 1}, \psi_{g, 1}^{\text {inc }}\right), \ldots,\left(\sigma_{g}^{-1} q_{g, M}, \psi_{g, M}^{\text {inc }}\right)\right)
\end{gathered}
$$

Letting $\psi_{g} \equiv\left(\psi_{g, 1}, \ldots, \psi_{g, M}\right)$ and dividing by $\sigma_{g},(4)$ can be written more compactly in operator notation as

$$
\mathcal{H}_{g} \psi_{g}-\mathcal{Z} \mathcal{L}^{+} \sum_{g^{\prime}=1}^{G} \Gamma_{g, g^{\prime}} \mathcal{L} \psi_{g^{\prime}}=\mathcal{Q}_{g}, \quad 1 \leq g \leq G .
$$

The discretization of (1) is completed by applying a spatial discretization to (12). For simplicity, we assume that the spatial domain $D$ is a three-dimensional rectangular domain partitioned into a Cartesian grid of "zones". Upon this grid, any one of a variety of discretization schemes can be implemented, such as diamond-differencing, discontinuous finite element methods or modifications of these. For our present purposes, the particular choice of spatial discretization is irrelevant provided that it satisfies two conditions. First, we require that the discrete analogs of the functions $\psi_{g, m}$ can be represented by a vector $\Psi_{g, m}$ of length $N$, where $N$ is proportional to the number of zones. The discrete analog of the unknown $\psi_{g}$ in (12) is then $\Psi_{g} \equiv\left(\Psi_{g, 1}, \ldots, \Psi_{g, M}\right)^{T}$, and matrices and vect. $\quad H_{g, m}, H_{g}, L, L^{+}, \Gamma_{g, g^{\prime}}, Z_{m}, Z, Q_{g}$ corresponding w. the continuous operators $\mathcal{H}_{g, m}, \mathcal{H}_{g}, \mathcal{L}, \mathcal{L}^{+}, \Gamma_{g, g^{\prime}}$, $\mathcal{Z}_{m}, \mathcal{Z}, \mathcal{Q}_{g}$ can be defined, respectively. The fully discrete analog of (12) therefore becomes

$$
H_{g} \Psi_{g}-Z L^{+} \sum_{g^{\prime}=1}^{G} \Gamma_{g, g^{\prime}} L \Psi_{g^{\prime}}=Q_{g}, \quad 1 \leq g \leq G .
$$

The second condition we require of the spatial discretization is that the zones can be enumerated (along with the corresponding entries in the matrix and vector objects in (13)) in such a way that $H_{g, m}$ is a block lower triangular matrix such that, given any $N$-vector $v$, the product $H_{g, m}^{-1} Z_{m} v$ can be computed using at most $\chi_{\mathrm{c}} N$ floating point operations storing at most $\chi_{m} N$ floating point numbers (not including the memory required for $v$ ). The proportionality constants $\chi_{m}$ and $\chi_{c}$ depend upon the spatial discretization. In the common terminology of the numerical transport community, a more familiar way of expressing this second 
condition is that $H_{g, m}^{-1} Z_{m}$ can be applied to a vector by performing a "transport sweep" of the spatial grid in the direction $\Omega_{m}$ with a computational complexity proportional to the number of zones. Standard spatial discretizations such diamond differencing or discontinuous finite element methods satisfy both of these properties.

Equation (13) is a $G M N$-dimensional linear system that is sometimes referred to as the flux formulation. A lower-dimensional formulation can be obtained by considering $\Phi_{g} \equiv L \Psi_{g}$ as the unknowns. This $G N$ dimensional system is called the moment formulation or the integral equation formulation, since $L \Psi_{g}$ represents the discretized first moment of $\Psi_{g}$ with respect to the direction variable $\Omega$. This formulation is obtaired by premultiplication of (13) by $\mathrm{LH}_{g}^{-1}$, which yields, for $g=1, \ldots, G$,

$$
\Phi_{g}-K_{g} \sum_{g^{\prime}=1}^{G} \Gamma_{g, g^{\prime}} \Phi_{g^{\prime}}=L H_{g}^{-1} Q_{g},
$$

where

$$
K_{g} \equiv L H_{g}^{-1} Z L^{+}
$$

This can be written in matrix form as

$$
A \Phi=R,
$$

where

$$
\begin{aligned}
A & \equiv I-K \Gamma \\
& \equiv\left(\begin{array}{ccc}
I & & \\
& \ddots & \\
& & I
\end{array}\right) \\
& -\left(\begin{array}{cccc}
K_{1} & & \\
& \ddots & \\
& & K_{G}
\end{array}\right)\left(\begin{array}{ccc}
\Gamma_{1,1} & \cdots & \Gamma_{1, G} \\
\vdots & & \vdots \\
\Gamma_{G, 1} & \cdots & \Gamma_{G, G}
\end{array}\right)
\end{aligned}
$$

and

$$
\Phi \equiv\left(\begin{array}{c}
\Phi_{1} \\
\vdots \\
\Phi_{G}
\end{array}\right), \quad R \equiv\left(\begin{array}{c}
L H_{1}^{-1} Q_{1} \\
\vdots \\
L H_{G}^{-1} Q_{G}
\end{array}\right)
$$

By applying $H_{g}^{-1}$ to (13), we obtain

$$
\Psi_{g}=H_{g}^{-1}\left(Z L^{+} \sum_{g^{\prime}=1}^{G} \Gamma_{g, g^{\prime}} \boldsymbol{\Phi}_{g^{\prime}}+Q_{g}\right) .
$$

Thus, the solution of (13) can be recovered from the solution of the linear system (16).

\section{An algorithm for the concurrent so- lution of the linear system}

The matrix $A$ defined by (17) is dense and, for most practical problems, is of sufficiently large order that it is infeasible to explicitly compute and store its entries. The system (16) must therefore be solved iteratively using a method that does not require the explicit availability of $A$. Fortunately, many iterative methods satisfy this requirement, the simplest example of which is Richardson iteration, in which the recursion

$$
\Phi_{k+1}=(I-A) \Phi_{k}+R
$$

is performed until some convergence criteria is met involving the difference $\Phi_{k+1}-\Phi_{k}$, which is the residual of (16) corresponding to the approximate solution $\Phi_{k}$. In fact, the iteration (20) is precisely the so-called "source iteration" algorithm used in many neutron transport codes. This iteration represents the procedure of taking an initial guess of the vector $\boldsymbol{\Phi}=L \Psi$, evaluating the scattering term (involving $\Gamma$ ) as a source term for the subsequent inversion (via transport sweeps over the spatial grid) of the differential operator represented by $H$ to obtain $\Psi$, which is then integrated over all directions to obtain an updated vector $\Phi$.

The rate of convergence of the iteration (20) is completely determined by the spectral radius of the matrix $I-A$, which must be less than unity. For problems in which all of the $\sigma_{g}$ are comparable to or smaller than the diameter of the spatial domain $D$, this spectral radius is sufficiently small that the iteration converges in a few iterations. For other problems, it is advantageous to consider iterative methods that are more efficient than (20) (such as adaptive Chebyshev and Generalized Minimum Residual (GMRES)) as well as preconditioning, or "acceleration", strategies $[3,4]$. As with the simpler Richardson iteration, these advanced methods share the common feature that the only information required about the system matrix $A$ is how to multiply it times a vector (a characteristic of Krylov subspace methods). It is also the case that this matrixvector multiplication step is the dominant cost per iteration in all of these methods. We therefore focus exclusively on this step in the remainder of the paper.

\subsection{Scalability requirements}

Our goal is to design a concurrent algorithm for the solution of (16) that is scalable with respect to each of the phase space variables $G, M$ and $N$. Ideal scalability would mean that, if our algorithm requires $T$ 
time units to solve (16) for specific values of $G, M$ and $N$ on some number $\rho$ of nodes sufficient to store the problem in their aggregate memory, then the system (16) resulting from an increase of any or all of these phase space variables can still be solved in $T$ time units and stored in the aggregate nodal memory of $\alpha \rho$ nodes, where $\alpha$ is proportional to the increase in memory requirement and computational complexity. Due to communication costs and the fact that, in general, the number of iterations required to solve (16) to a given tolerance will not remain constant as the problem size grows, the scalability represented in the "constant $T$ " definition given above is not practically possible. Instead, we will be satisfied with an algorithm that limits the growth in the time to perform the matrix-vector product $A \Phi$ to, at worst, a logarithmic function of the phase space variables.

The formation and iterative solution of the problem (16) requires sufficient memory to store the $G N$-vector $R$, the $G N$-vectors generated during the iteration, as well as the data necessary to compute the product of $A$ times an arbitrary $G N$-vector. Given a vector $\Phi$ of length $G N$, the formation of the product $A \Phi$ requires (i) the multiplication of $\Phi$ by the matrix $\Gamma$, (ii) the multiplication of $\Gamma \Phi$ by $K$, and (iii) the subtraction of $K \Gamma \Phi$ from $\Phi$. We now consider the sequential memory requirement and floating point complexity of each of these steps to arrive at totals for the product $A \Phi$.

Memory requirement. The submatrices $\Gamma_{g, g^{\prime}}$ of $\Gamma$ are diagonal matrices of dimension $N$, so, in general, the memory required to store the non-zero entries of $\Gamma$ is $G^{2} N$ floating point numbers. The matrix $L^{+}$ is simply an injection with no memory requirements, and the matrix $L$ requires only the storage of the $M$ quadrature weights $w_{m}$. From the assumptions made in Section 2, the memory required to evaluate the product of the matrix $H_{g, m}^{-1} Z_{m}$ with a vector of length $N$ is at most $\chi_{m} N$ for some number $\chi_{m}$ depending upon the spatial discretization. Thus, the memory required to multiply $K$ times a vector is $\chi_{m} G M N+M$. The memory required to multiply $A$ times a vector is therefore $G\left(G+\chi_{m} M\right) N+M$.

Floating point complexity. As mentioned above, each submatrix $\Gamma_{g, g^{\prime}}$ is a diagonal matrix of dimension $N$. Thus, the number of floating point operations required to compute $\Gamma \Phi$ is $G(2 G-1) N$. From (15), the multiplication of $K_{g}$ times a vector of length $N$ involves multiplication by $L^{+}$(which requires no floating point operations) followed by a multiplication by $H_{g}^{-1} Z$ (which requires $\chi_{c} N$ floating point operations by assumption) followed by a multiplication by $L$ (which requires $(2 M-1) N$ floating point op-

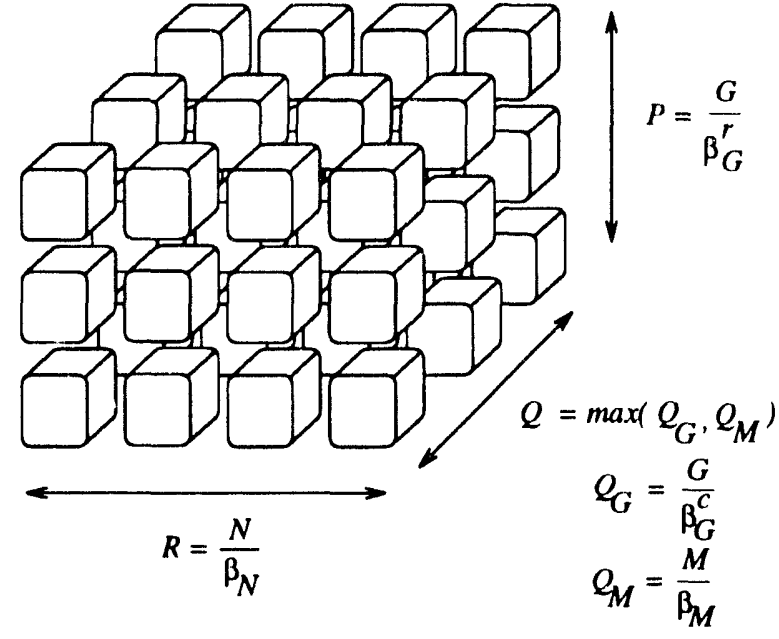

Figure 1: Virtual $P \times Q \times R$ nodal grid.

erations). Thus, the number of floating point operations required to multiply the block diagonal matrix $K$ times the vector $\Gamma \Phi$ is $G\left(\chi_{c}+2 M-1\right) N$. Given $K \Gamma \Phi$, the computation of $\Phi-K \Gamma \Phi$ requires $G N$ floating point operations, so the total number of floating point operations required to compute $A \Phi$ is therefore $G(2 G-1) N+G\left(\chi_{c}+2 M-1\right) N+G N=$ $G\left(2(G+M)+\chi_{c}-1\right) N$.

From the preceding computations, we therefore see that both the sequential memory requirement and computational complexity can be modeled as a trilinear function of variables $P, Q$ and $R$ where $P$ is proportional to $G, R$ is proportional to $N$, and $Q$ is proportional to $G$ and/or $M$. This observation motivates our data layout strategy, in which we imagine the available nodes virtually organized as a $P \times Q \times R$ nodal grid (see Figure 1) where

- each $Q R$ node plane is assigned to a unique block of $\beta_{G}^{r}$ energy groups, which implies that $P=G / \beta_{G}^{r}$,

- each $P R$ node plane is assigned to a unique block of $\beta_{G}^{c}$ energy groups and/or $\beta_{M}$ directions so that $Q=\max \left(Q_{G}, Q_{M}\right)$ where $Q_{G}=G / \beta_{G}^{c}$ and $Q_{M}=M / \beta_{M}$,

- each $P Q$ node plane is assigned to a unique block of $\beta_{N}$ spatial unknowns, which implies that $R=$ $N / \beta_{N}$.

In saying that a nodal plane is "assigned" to a block of unknowns, we mean that each node of the plane contains all data corresponding to that block of unknowns and performs all operations using this data. 

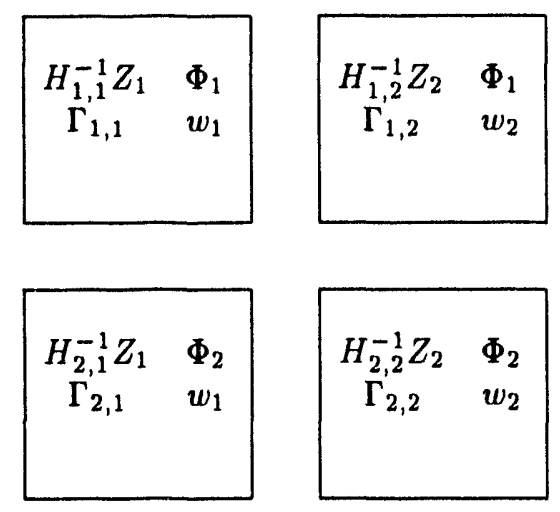

Figure 2: Initial data layout.

In the following two subsections, we describe the data layout and communication strategy in more detail.

\subsection{Concurrency in energy and direction}

To simplify the description of the concurrent application of $A$ to an arbitrary vector $\Phi$, we begin with the special case in which $R=1$ so that the nodal grid can be considered as simply a $P \times Q$ array. That is to say, we will ignore spatial concurrency and consider only concurrency in energy and direction. In our example, we assume that $G=M=2$ and we imagine four nodes arranged in a logical $2 \times 2$ grid $(P=Q=2$ ) with data preloaded as shown in Figure 2. For $p=1,2$ and $q=1,2$, the $(p, q)$-th node of the array contains the data necessary to compute the product of $H_{p, q}^{-1} Z_{q}$ times a vector for group $p$ and direction $q$. The $(p, q)$-th node also contains the submatrix $\Gamma_{p, q}$ coupling groups $p$ and $q$. Note here that the in$\operatorname{dex} q$ has referenced both group and direction indices, which is a result of our intent to have $Q$ accommodate concurrency in both energy and direction. The vector $\Phi=\left(\Phi_{1}, \Phi_{2}\right)^{T}$ is distributed across the nodal grid rows and replicated the columns. The quadrature weights $u_{1}$ and $w_{2}$ are distributed across nodal grid columns and replicated across the rows. Given this data distribution, the product $\bar{\Phi}=A \Phi$ is computed by the following sequence of six steps. The product vector $\bar{\Phi}$ thereby obtained will have the same distribution as $\Phi$.

Step 1: Transpose $\Phi$ in the nodal grid. See Figure 3.

Step 2: In each node, multiply the submatrices $\Gamma_{g, g^{\prime}}$ of $\Gamma$ against their respective subvectors $\Phi_{g^{\prime}}$ of $\Phi$, overwriting the transposed $\Phi$. See Figure 4 .

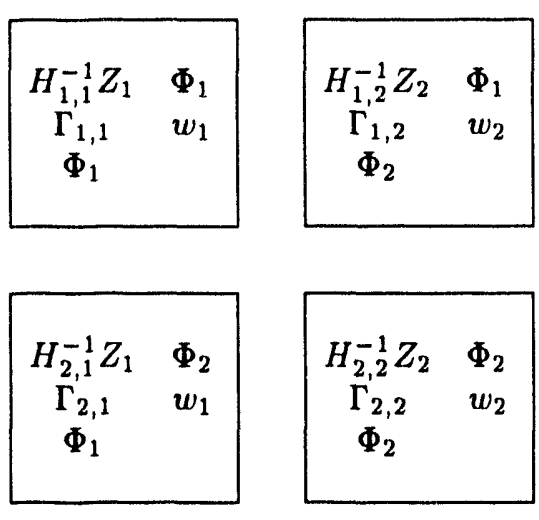

Figure 3: Step 1.

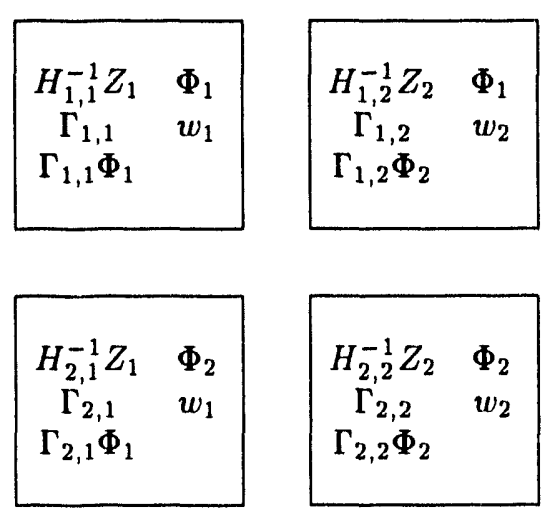

Figure 4: Step 2.

Step 3: Complete the product $\Gamma \Phi$ by combining the partial sums $\Gamma_{g, g^{\prime}} \Phi_{g^{\prime}}$ across the columns of the nodal grid. That is, the two nodes in each row exchange their local values of $\Gamma_{g, g^{\prime}} \Phi_{g^{\prime}}$ and accumulate. The result of this operation is that the product vector $(\Gamma \Phi)=$ $\left((\Gamma \Phi)_{1},(\Gamma \Phi)_{2}\right)^{T}$ is distributed across rows of the nodal grid and replicated across the columns. See Figure 5.

Step 4: Begin the multiplication of $(\Gamma \Phi)$ by $K$. Since $K$ is block diagonal, this means that we must compute $K_{g}(\Gamma \Phi)_{g}=L H_{g}^{-1} Z L^{+}(\Gamma \Phi)_{g}$ for $g=1,2$. The function of the operator $L^{+}$applied to a vector $(\Gamma \Phi)_{g}$ is to replicate it $M=2$ times. Note, however that the combine operation that concluded the previous step has already performed this replication across the nodal grid columns. From (5), we see that the application of $H_{g}^{-1} Z$ can be accomplished in parallel over the directions $m=1,2$. Due to our a priori distribution of 

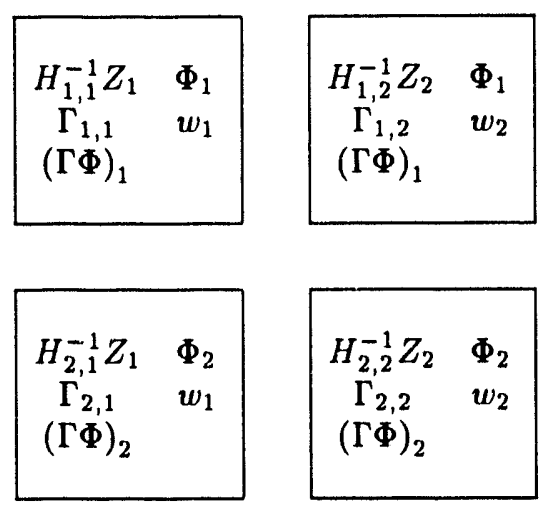

Figure 5: Step 3.

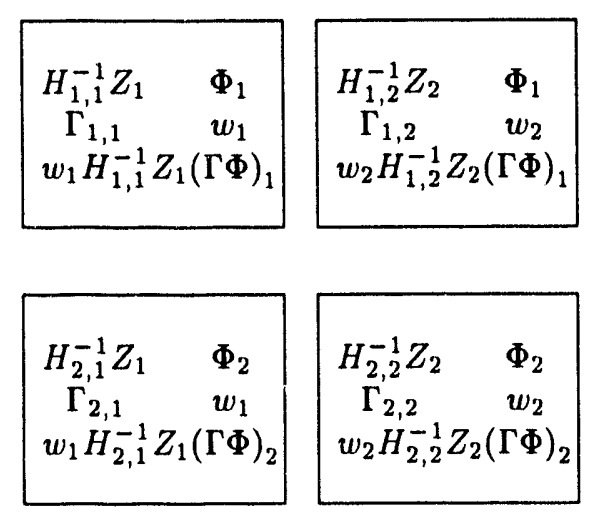

Figure 6: Step 4.

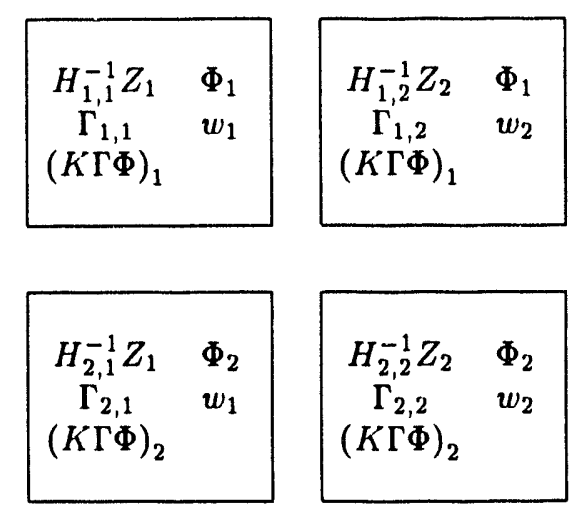

Figure 7: Step 5.

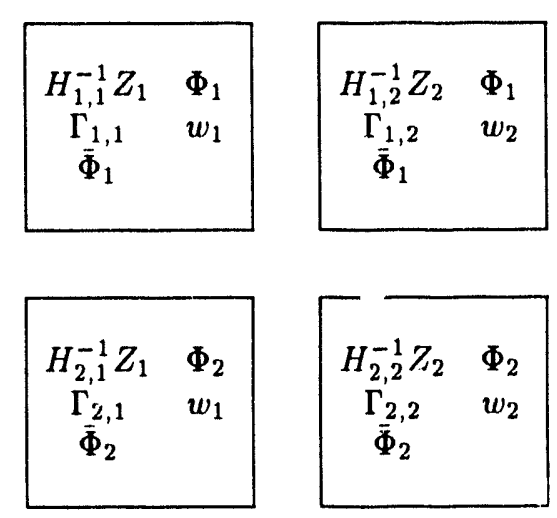

Figure 8: Step 6.

the $H_{g, m}^{-1} Z_{m}$, this step can be done solely with local computation. In anticipation of applying the operator $L$, we also multiply by the quadrature weights $w_{m}$ in each rode. See Figure 6.

Step 5: Complete the action of the operator $L$ by combining the quantities $w_{m} H_{g, m}^{-1} Z_{m}(\Gamma \Phi)_{g}$ in each nodal grid row to obtain the vector $(K \Gamma \Phi)=$ $\left((K \Gamma \Phi)_{1},(K \Gamma \Phi)_{2}\right)^{T}$ distributed across the rows of the nodal grid and replicated across the columns. See Figure 7.

Step 6: Complete the computation of $\bar{\Phi}=$ $\left(\bar{\Phi}_{1}, \bar{\Phi}_{2}\right)^{T}=A \Phi$ by forming $\bar{\Phi}_{g}=\Phi_{g}-(K \Gamma \Phi)_{g}$ in each node. The product $A \Phi$ now has the same data layout as the original vector $\Phi$. See Figure 8 .

In this example we assigned exactly one energy group or direction to a nodal grid row or column. As indicated earlier, we wish to allow for the assignment of multiple energy groups or directions to a single node through the use of the blocking factors $\beta_{G}^{r}, \beta_{G}^{c}$ and $\beta_{M}$ introduced above. The example given here readily generalizes to cases in which the blocking factors can be choseu so that $Q=Q_{G}=Q_{M}$ and $P$ and $Q$ are any positive integer.

The use of nodal grid columns to perform "doubleduty" in representing both group and direction concurrency allowed the same nodal grid to be used to accommodate the concurrent multiplication of a vector by $\Gamma$ and by $K$. In addition to nceding $Q$ to be proportional to $G$ and/or $M$ as described in the scalability discussion above, we see that this strategy leads to a serendipitous pipelining at the point where the operator $L^{+}$is applied ( $L^{+}$is "no-op" rather than a broadcast).

For cases for which $G \neq M$, or for which blocking 
factors $\beta_{G}^{c}$ and $\beta_{M}$ cannot be found so that $Q_{G}=Q_{M}$ in the general case, the algorithm described above can still be used, although some nodes will be idle during certain steps. If $Q_{G}<Q_{M}$, then $Q_{M}-Q_{G}$ nodal grid columns will be idle during Steps 1 through 3 and 6 , which will involve only a $P \times Q_{G}$ subgrid of the $P \times Q$ grid. If $Q_{M}<Q_{G}$, then $Q_{G}-Q_{M}$ nodal grid columns will be idle during Steps 4 and 5 , which will involve only a $P \times Q_{M}$ subgrid. Given that the amount of floating point computation required to perform Steps 4 and 5 will always dominate that required for the other steps, it is preferable to have $Q_{G} \leq Q_{M}$ if at all possible. In any case, we remark that the existence of the load imbalance resulting from idle nodes does not preclude a scalable algorithm. We can tolerate a certain amount of the inefficiency provided that it does not significantly increase with the problem size and number of nodes.

\subsection{Spatial concurrency}

We now consider the exploitation of spatial concurrency in the coniputation of $A \Phi$. As described above, this concurrency will be accommodated using the $R$ axis of the virtual $P \times Q \times R$ nodal grid.

Recalling our assumption that the spatial domain $D$ is a three-dimensional rectangular solid that is partitioned into a Cartesian grid of spatial zones, we further assume that the grid is partitioned into a Cartesian grid of subdomains. Each subdomain therefore consists of a Cartesian grid of spatial zones. For simplicity, we assume that all subdomains contain the same number of spatial zones. Let $R$ be the number of subdomains, and let $\beta_{N}$ denote the number of spatial unknowns per subdomain.

As described above, each $P Q$ plane of the $P \times Q \times R$ nodal grid is assigned to a unicue subdomain. We would like to apply the algorithm described in the preceding subsection (for the special case $R=1$ ) as if the problems on each subdomain were independent. With the exception of the multiplication of the matrix $H_{g}^{-1} Z$ times an $N$-vector distributed across the $P Q$ grid planes, there is no coupling of the spatial unknowns in the computation of $A \Phi$. Therefore, in Steps $1-3,5$ and 6 of the algorithm described in the preceding section (replicated in each $P Q$ plane), there is in fact no communication among the $P Q$ nodal grid planes. In Step 4, however, the multiplication of $\mathrm{H}_{g}^{-1} \mathrm{Z}$ times a vector corresponds to solving a set of discrete, firstorder linear hyperbolic equations on the entire spatial grid, and therefore does require communication among the $P Q$ nodal grid planes. We consider this computation in more detail.

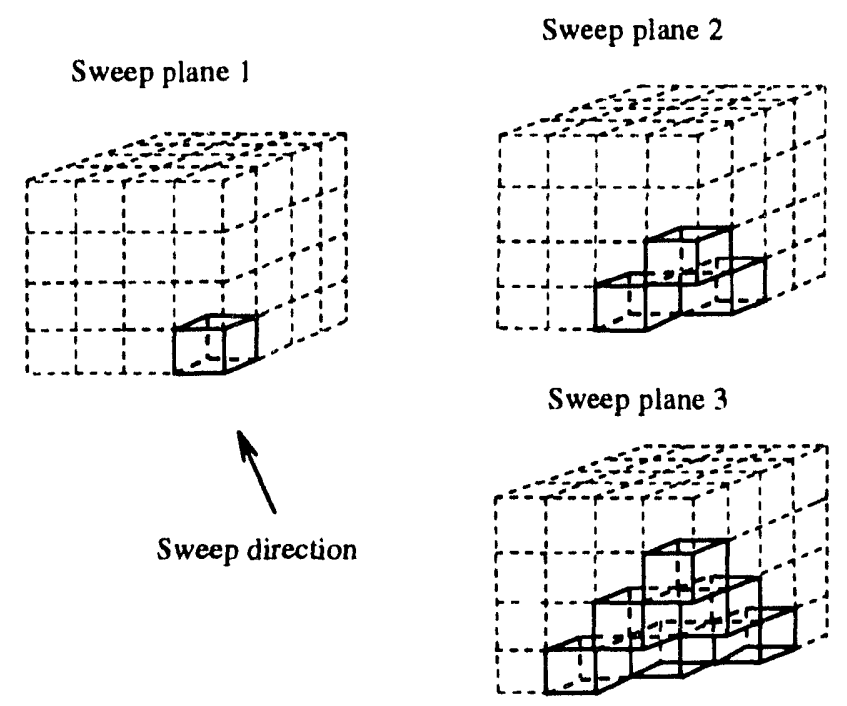

Figure 9: Grid sweeping by subdomains.

The multiplication of $\mathrm{H}_{g}^{-1} \mathrm{Z}$ times a vector $v$ can be performed by independently and concurrently computing $H_{g, m}^{-1} Z_{m} v_{g, m}$ where $v_{g, m}$ are subvectors of $v$, each of which contains quantities corresponding to the entire spatial grid. The computation of $H_{g, m}^{-1} Z_{m} v_{g, m}$ can therefore proceed asynchronously within each $R$ line (a set of nodes in the grid with the same $P$ and $Q$ coordinates). Since, by assumption, each $H_{g, m}$ is a block lower triangular matrix, $H_{g, m}^{-1} Z_{m} v_{g, m}$ is computed by forward elimination, which can also be viewed as performing "transport sweeps" across subdomains in the direction given by $\Omega_{m}$ starting with boundary conditions given on the three faces of $D$ on which $\Omega_{m}$ points into the domain. Given the partitioning of the spatial grid into a Cartesian grid of subdomains, these sweeps can be performed subdomain by subdomain in the direction $\Omega_{m}$. Within a subdomain, the angular fluxes on the three upstream (relative to $\Omega_{m}$ ) faces are computed by, and received in a message from, nodes responsible for the three subdomains sharing these faces. The subdomain grid is then swept in the direction $\Omega_{m}$ (i.e., the block of $H_{g, m}$ corresponding to the current subdomain is inverted by forward elimination) producing angular fluxes on the downstream faces, which are then sent in a message to the nodes responsible for the three subdomains sharing these faces. This sweeping by subdomains logically proceeds as a sequence of sweeps along "planes" of subdomains, as depicted in Figure 9. To avoid unnecessary synchronization, it preferable to have the nodes operate reactively during this part of Step 4, rather than in strict lock step as suggested in Figure 9. 


\section{An implementation}

We have created a code, named $A r d r a$, to form and solve (16) using the algorithm described in Section 3. Ardra is written in the $\mathrm{C}$ language. It is layered upon Zipcode $[5,6]$, which is a message-passing communications library supported on many distributed memory platforms including the nCUBE/2, the Intel Gamma and Delta Prototypes and Paragon, and the Thinking Machines CM-5. It is also supported on homogeneous clusters of Sun Sparcstations and IBM RS6000 workstations. Therefore, Ardra runs on all of these platforms as well.

In addition to the portability it provides, Zipcode supports the three-dimensional nodal grid abstractions we have used to conceptualize the algorithm described in Section 3. Specifically, a $P \times Q \times R$ nodal grid can be created in Zipcode, and various communication operations required by the algorithm are provided relative to this grid and all subgrids thereof. In fact, each of the communication opera. ons contained in the algorithm of Section 3 essentially corresponds to a single Zipcode primitive. These features of Zipcode, in addition to its handling of the mapping of virtual to physical nodes and interfacing to vendor-provided message passing syntax, enable Ardra's structure to closely mimick the abstract description of the algorithm presented in Section 3.

\section{Numerical results}

Using Ardra, we examined the scalability of our algorithm and the performance of the code on the 1024node $\mathrm{nCUBE} / 2$ at the Massively Parallel Computing Research Laboratory (MPCRL) at Sandia National Laboratory. All of the results reported below are for the computation of $A \Phi$ only using the algorithm described in Section 3. The cost of this operation will dominate the total cost per iteration of solving (16) using iterative methods such as Richardson, adaptive Chebyshev or Generalized Minimum Residual (GMRES). Therefore, in these tests the numerical values of problem-dependent quantities such as $\Gamma$ or the physical dimensions of the spatial domain are irrelevant, since the values of these variables only affect the number of iterations required, not the cost per iteration. The latter cost is determined entirely by the number of groups $G$, the number of directions $M$, and the number of spatial unknowns $N$, i.c., the phase space dimensions.

In reporting the number of megaflops (Mflops) required to form $A \Phi$, we count only sequential floating point operations. That is, we only count the number of floating point operations that would have been required had the operation been performed on a sequential machine. This number could be substantially less than the number of floating point operations actually performed by the machine. For example, in performing a combine operation with a recursive doubling algorithm (as is implemented in the combine operations in Zipcode), scalability is achieved by performing some redundant computation to reduce communication. However, we do not count such redundancies in our floating point operation count. To do so would be to disguise communication costs as useful floating point work.

The times reported for the computation of $A \Phi$ are averages of the times to compute $A \Phi$ in ten successive iterations of Richardson iteration applied to the solution of (16). On the nCUBE/2, the variation of times from iteration to iteration was negligible, so there was no need to compute, for example, a standard deviation.

\subsection{Scalability in energy and directıon in the ideal case}

Our first test was to determine how well the algorithm (and implementation) scales with respect to an increasing number of energy groups $G$ and number of directions $M$ in the ideal case where $Q_{G}=$ $Q_{M}=Q$. For all of the problems in this test suite, $\beta_{G}^{r}=\beta_{G}^{c}=1$ (i.e., one energy group per nodal grid row and column), and $\beta_{M}=4$ (i.e., 4 directions per nodal grid column). This, together with the constraint $Q_{G}=Q_{M}=Q$, implies that $P=G=M / 4=Q$. The spatial domain was a $20 \times 20 \times 20$ cube so that $N=8000$. No spatial decomposition was employed (i.e., $R=1$ ). Under these assumptions, we performed $S_{2}, S_{4}, S_{6}, S_{8}$ and $S_{10}$ calculations by setting $M=8,24,48,80$ and 120 , respectively. Since the number of spatial unknowns $N$ and the blocking factors $\beta_{G}^{r}, \beta_{G}^{c}$, and $\beta_{M}$ were held fixed, the problem size and work per node was the same for all five problems. Therefore, in this test suite the problem size was increased in direct proportion to the number of nodes $\rho=P Q=4,36,144,400$, and 900 .

Table 1 summarizes the results obtained for the five problems. The main result of interest is the fact that the time to compute $A \Phi$ increased only very slightly as the number of nodes (and problem size) were increased. This bodes well for the ability to solve even larger problems on a larger number of nodes in roughly the same amount of time, which is our functional definition of scalability. We also note that we nearly 


\begin{tabular}{|c|c|c|c|c|}
\hline $\begin{array}{c}\text { Groups } \\
G\end{array}$ & $\begin{array}{c}\text { Dirs. } \\
M\end{array}$ & $\begin{array}{c}\text { Grid } \\
(P, Q, R)\end{array}$ & $\begin{array}{c}\text { Time } \\
(\text { sec. })\end{array}$ & $\begin{array}{c}\text { Mflops } \\
\text { per sec. }\end{array}$ \\
\hline 2 & 8 & $(2,2,1)$ & 1.4109 & 4.9 \\
\hline 6 & 24 & $(6,6,1)$ & 1.5545 & 39.9 \\
\hline 12 & 48 & $(12,12,1)$ & 1.6454 & 150.8 \\
\hline 20 & 80 & $(20,20,1)$ & 1.7369 & 396.9 \\
\hline 30 & 120 & $(30,30,1)$ & 1.6938 & 915.7 \\
\hline
\end{tabular}

Table 1: Performance results for the ideal case.

achieved a gigaflop per second on the last problem, which is roughly half the peak speed of the nCUBE/2.

\subsection{Scalability in energy}

Our second set of problems tests the scalability of the algorithm with respect to the number of groups $G$ alone. Specifically, the number of directions $M$ is held fixed at $M=8,24,48,80$, or 120 while the number of groups $G$ and the number of nodes $\rho$ are increased. The spatial domain is again a $20 \times 20 \times 20$ cube, and no spatial decomposition is used (i.e., $N=8000$ and $R=1$ ). In all cases, $\beta_{G}^{r}=\beta_{G}^{c}=1$, assigning one energy group to each nodal grid row and column, and the blocking factor $\beta_{M}$ is chosen to be the smallest divisor of $M$ satisfying $Q_{M} \equiv M / \beta_{M} \leq Q_{G}=G=Q$. The nodal grids are therefore square for each problem, and the rows and columns completely exhaust group concurrency. For $M$ fixed, as $G$ and $\rho=P Q=G^{2}$ increase, the blocking factor $\beta_{M}$ will eventually tee set to 1 . Beyond this point (i.e., for larger values of $G$ and $\rho$ ), the number of nodal grid columns exceeds the concurrency in direction, so some nodes will be idle during a portion of the computation of $\boldsymbol{A} \boldsymbol{\Phi}$.

Figure 10 displays the results for this test suite. Each of the five curves shown corresponds to a set of runs with $M$ fixed and $G=1,2, \ldots$ In all five cases, the time to compute $A \Phi$ tends to a fairly constant value as the number of groups $G$ and the number of nodes $\rho=P Q=G^{2}$ is increased.

\subsection{Scalability in direction}

Our third set of problems tests the scalability of the algorithm with respect to the number of directions $M$, holding the number of groups $G$ fixed. For all problems, we set $\beta_{G}^{r}=1$ (one energy group per nodal grid row), $\beta_{M}=1$ (one direction per nodal grid column) and take $\beta_{G}^{c}$ (the number of energy groups assigned to each nodal grid column) to be the smallest divisor of $G$ such that $Q_{G}=G / \beta_{G}^{\mathrm{c}} \leq Q_{M}=M=Q$.

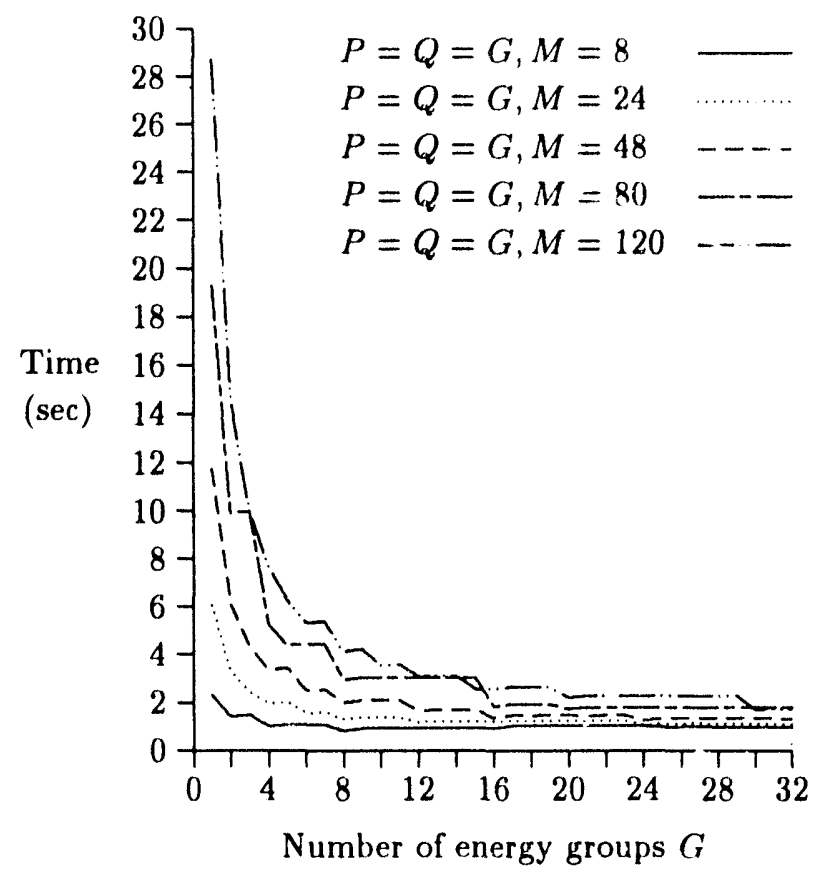

Figure 10: Scalability of time with respect to an increasing number of energy groups.

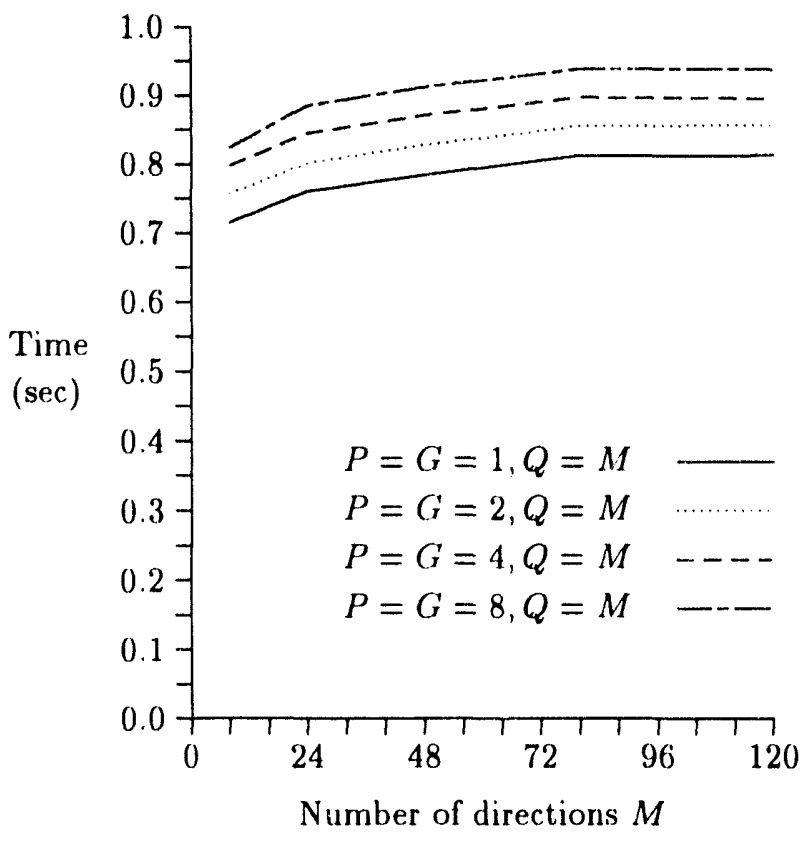

Figure 11: Scalability of time with respect to an increasing number of directions. 


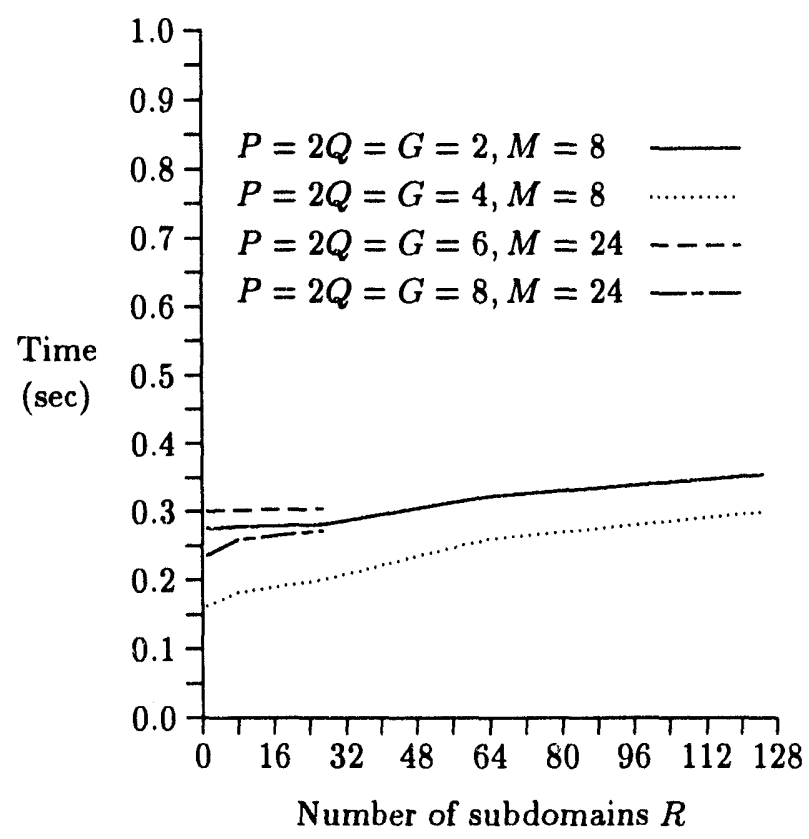

Figure 12: Scalability of time with respect to an increasing number of subdomains.

The spatial domain is again a $20 \times 20 \times 20$ cube, and no spatial decomposition is used (i.e., $N=8000$ and $R=1$ ). Figure 11 displays the resulting timing measurements. Each curve was generated by fixing the number of groups at $G=1,2,4$ or 8 while increasing the number of directions $M$ and number of nodes $\rho=P Q=G M$, using the blocking factors described above. Figure 11 shows that the time to compute $A \Phi$ again increases only very slowly in the limit of large $M$. Since the number of spatial unknowns $N$ and the blocking factors $\beta_{G}^{r}, \beta_{M}$ and $\beta_{G}^{c}$ (for $M \geq G$ ) are fixed, the work per node is constant in this limit.

\subsection{Scalability in space}

Our fourth and final set of problems tests scalability with respect to the number of spatial subdomains $R$. There are four problems in this set corresponding to $G=2,4,6$ and 8 . For all problems, $\beta_{G}^{r}=1$ and $\beta_{G}^{c}=2$, which implies that $P=2 Q=G$. The number of directions $M$ is 8 for the first two problems and 24 for the second two. The blocking factors $\beta_{M}$ were 8 , 4,8 and 6 , respectively, so that $Q=Q_{G}=Q_{M}$ for all problems (the ideal case). For each of the four problems, a subdomain consists of a $10 \times 10 \times 10$ array of zones $\left(\beta_{N}=1000\right)$, and we hold all quantities fixed except for the number of subdomains $R$, which we in- creased until no further nodes were available. Given the fact that $P=2 Q=G$ for all problems in this set, $R$ can be at most $2048 / G^{2}(=1024 / P Q)$ on the 1024 node nCUBE/2. The results shown in Figure 12 indicate a iairly slow rate of increase in the matrix-vector multiplication time as the number of subdomains, and therefore, number of spatial unknowns in the problem, is increased.

\section{Acknowledgement}

We thank the Massively Parallel Computing Research Laboratory at Sandia National Laboratory in Albuquerque for providing access to their $\mathrm{nCUBE} / 2$.

\section{References}

[1] G. I. Bell and S. Glasstone, Nuclear Reactor Theory, Van Nostrand, New York, 1970.

[2] G. I. Marchuk and V. I. Lebedev, Numerical Methods in the Theory of Neutron Transport, Harwood Academic Publishers, Chur, 1986.

[3] S. F. Ashby, P. N. Brown, M. R. Dorr and A. C. Hindmarsh, "Preconditioned Iterative Methods for Discretized Transport Equations", in Proc. International Topical Meeting of the American Nuclear Society-Advances in Mathematics, Computations, and Reactor Physics, Volume 2, American Nuclear Society, April 1991, pp. 6.1 2-1 to 6.129. Also Lawrence Livermore National Laboratory Technical Report UCRL-JC-104901, July 1990.

[4] S. F. Ashby, P. N. Brown, M. R. Dorr and A. C. Hindmarsh, "A Linear Algebraic Analysis of Diffusion Synthetic Acceleration for the Boltzmann Transport Equation", Lawrence Livermore National Laboratory Report UCRL-JC-110712, May 1992, to appear in SIAM J. Numer. Anal.

[5] A. Skjellum and M. Morari, "Zipcode: A Portable Communication Layer for High Performance Multicomputing-Practice and Experience", Tech. Report UCRL-JC-106725, Lawrence Livermore National Laboratory, March 1991. To appear in Concurrency: Practice \& Experience.

[6] A. Skjellum and C. H. Still, "Zipcode: and the Reactive Kernel for the Caltech Intel Delta Prototype and nCUBE/2", in Proc. Sixth Distributed Memory Computing Conf. (DMCC'6), IEEE, April 1991, pp. 26-33. Also available as LLNL Technical Report UCRL-JC-107636. 

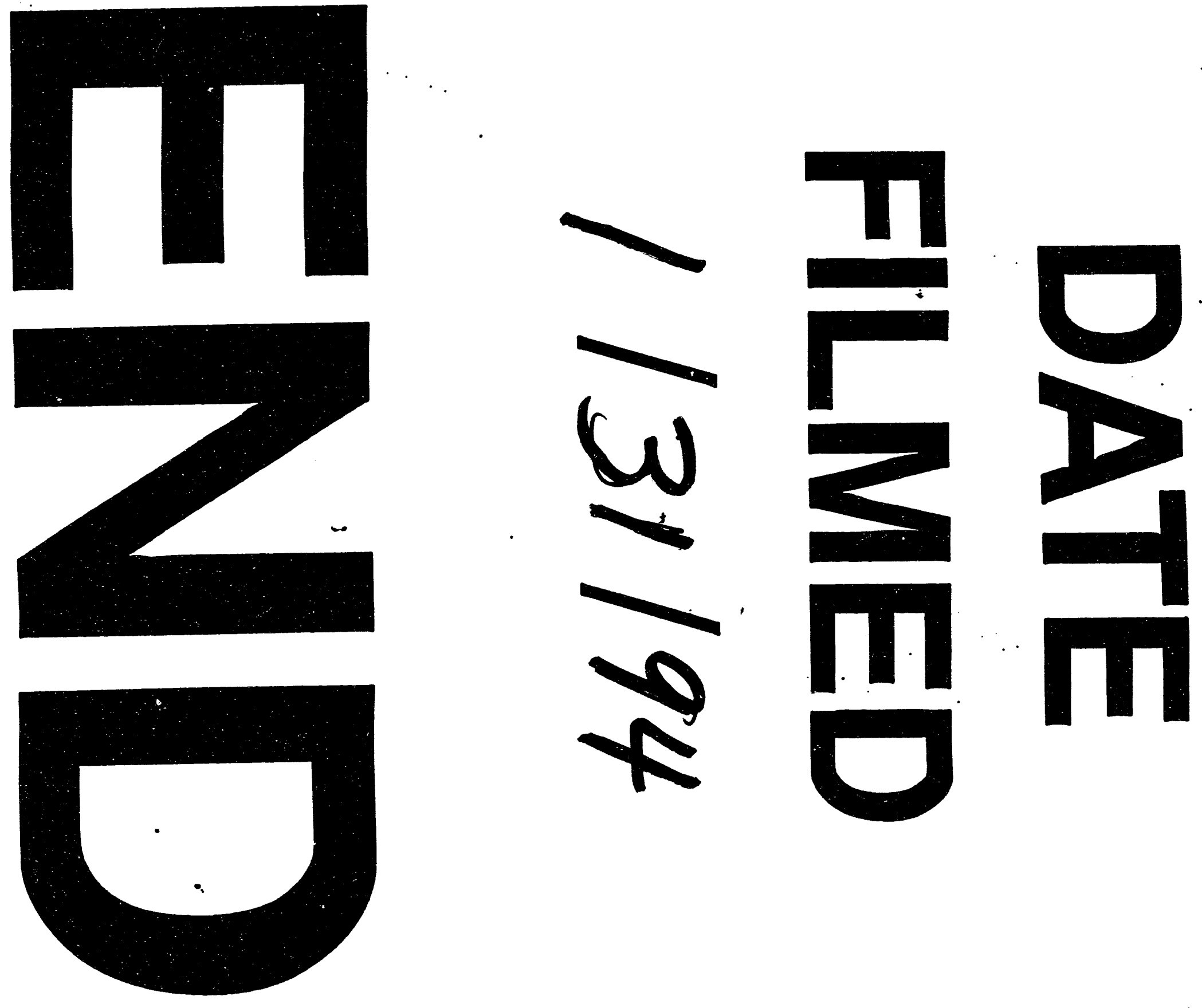
